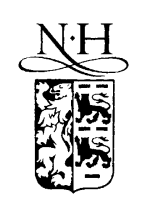

ELSEVIER

\title{
Time development during growth and relaxation of amorphous carbon films. Tight-binding molecular dynamics study
}

\author{
K. Kohary ${ }^{\text {a,b }}$, S. Kugler ${ }^{\text {a,* }}$ \\ a Department of Theoretical Physics, Budapest University of Technology and Economics, H-1521 Budapest, Hungary \\ ${ }^{\mathrm{b}}$ Fachbereich Physik und Zentrum für Materialwissenschaften der Philipps - Universität Marburg, D-35032 Marburg, Germany
}

\begin{abstract}
The growth of amorphous carbon (a-C) thin film on a [1 111$]$ diamond surface has been studied by a tight-binding (TB) molecular dynamics (MD) technique. Six different three-dimensional networks were constructed with periodic boundary conditions in two dimensions. Time-dependent non-equilibrium growth was simulated with atom-by-atom deposition and it was described as in real experiments without an artificial model of energy dissipation. An additional seventh structure was constructed by a melt quenching procedure which is widely used in computer generations of amorphous networks. The final structures consist of over 100 atoms. Densities, radial distribution functions (RDFs), coordination numbers, bond angle distributions and ring statistics were analyzed. During relaxation the temperature in the amorphous film decreases with stretched-exponential function. The time dependence of bond length and bond angle deviations were also investigated. (c) 2002 Elsevier Science B.V. All rights reserved.
\end{abstract}

PACS: $61.43 . \mathrm{Dq}$

\section{Introduction}

Carbon is one of the most exciting elements: $\mathrm{K}$ or $\mathrm{Rb}$ doped $\mathrm{C}_{60}$ show superconducting behavior, graphite is a good conductor, and it is also a good insulator in diamond form. Coexistence of $\mathrm{sp}^{2}$ and $\mathrm{sp}^{3}$ atomic sites can produce non-crystalline structures with a wide range of gap in the density of states. A neutron diffraction study of an amorphous carbon (a-C) sample prepared by a filtered vacuum-arc method, proposed almost one hundred

\footnotetext{
${ }^{*}$ Corresponding author. Tel.: +36-1 463 2146; fax: +36-1 463 3567.

E-mail addresses: krisztian.kohary@physik.uni-marburg.de (K. Kohary),kugler@phy.bme.hu (S. Kugler).
}

percent of $\mathrm{sp}^{3}$ atomic configuration (ta-C) [1]. A measurement on another a-C sample [2] showed mostly $\mathrm{sp}^{2}$ atomic arrangements. The $\mathrm{sp}^{3} / \mathrm{sp}^{2}$ ratio of bonding configuration is one of the most important parameter which determines whether it is a conductor, semiconductor or an insulator. The basic procedure for studying the atomic scale structures of non-crystalline solids is, so far, by the computer simulation. The diffraction measurement only reveals information about the structure in one dimension. There is, in addition, no experimental method for the determination of the microscopic arrangement in three dimensions. However, development in today's optics provides scientists with lasers of $\sim 10^{-15} \mathrm{~s}$ pulses. These can be used for the determination of local bonding configurations in the near future. 
Monte Carlo (MC) and molecular dynamics (MD) schemes are two main simulation methods. Recently, MC and Reverse MC schemes were applied for a-C $[3,4]$ providing large scale threedimensional models. MC simulations are very successful in finding the local minimal energy on an energy hypersurface, however dynamics of systems cannot be examined. Atomic scale modeling of the ion-beam-induced growth of a-C was simulated by Kaukonen and Nieminen [5] and by Jäger and Albe [6] using MD technique. Both works focused on the preparation of ta-C. They both applied classical empirical potentials $[7,8]$. A more accurate method is to derive the interatomic potential by quantum mechanical treatment. An approach called Car-Parrinello MD method [9] has been successfully applied for the preparation of a-C [10-12]. An alternative possibility to this method is to find less time-consuming tight-binding (TB) potentials that could be applied to larger systems (a few hundred atoms). In the last decade applications of different TB potentials were widespread in the MD study of a-C systems [13-18].

Our aim was to perform TB MD computer simulations to get thin amorphous films with a large number $(\sim 100)$ of atoms. We used the realistic TB Hamiltonian developed by $\mathrm{Xu}$ et al. [19] for carbon systems. The interatomic potential describes accurately the energetic, vibrational, and elastic properties of the diamond (fourfold), graphite (threefold), and linear-chain (twofold) structures in comparison with self-consistent firstprinciples density functional calculations. This realistic TB potential has already been used for the preparation of a-C $[13,18]$ and was successfully applied for the preparation of fullerenes [20]. In this paper we focus on the relaxation process after the growth and compare the results obtained by the melt quenching method.

\section{Simulation details}

Amorphous semiconductor films are usually prepared by atom-by-atom deposition techniques in the labs. During the sample preparation, single atoms or even small clusters move to the substrate. When they reach the surface they could (i) scatter backwards, (ii) penetrate under the surface atoms, (iii) start collision-cascade, or (iv) chemically bond on the surface producing the growth of a-C. The most decisive parameters for processes ((i)-(iv)) are substrate temperature $T_{\text {sub }}$ and the kinetic energies $E_{\text {beam }}$ of bombarding carbon atoms. The a-C is usually prepared at low pressure $\left(10^{-5}\right.$ mbar $)$, with the substrate temperature lower than $100{ }^{\circ} \mathrm{C}$. The aim of our computer simulation was to describe in detail the low energy $(1-5 \mathrm{eV})$ bombardment of the target surface. In this case the occurrence of processes (ii) and (iii) are highly negligible.

We have developed a TB MD computer code [21] to simulate the atom-by-atom deposition on a rectangular diamond a lattice substrate containing 120 carbon atoms. There were 24 fixed atoms at the bottom while the rest of the substrate atoms could move with full dynamics. The simulation cell was open along the [1 111 ] direction (positive $z$ axis). The kinetic energy of the atoms inside the substrate were rescaled at every time step $(\Delta t=$ $0.5 \mathrm{fs}$ ) in order to keep the substrate at a constant temperature. First, it was kept at a given temperature for $0.5 \mathrm{ps}$ to enable structural relaxation. In the deposition process the frequency of the atomic injection was on average $1 / 125 \mathrm{fs}^{-1}$. This flux is orders of magnitude higher than the deposition rate commonly applied in experiments. However, the low substrate temperatures (100 and $300 \mathrm{~K}$ ) during simulations cause quick energy dissipation and this may compensate the high deposition rate. Six different three-dimensional a-C networks have been constructed with periodic boundary conditions in two dimensions by this method [21].

Furthermore, an additional seventh model has been prepared by simulating a rapid cooling technique. In this case the temperature of the model e1 $T 100 L$ (see [21]) was increased up to $4200 \mathrm{~K}$. Considering this molten form of model e1 $T 100 \mathrm{~L}$, as an initial state, the trajectories of the atoms were followed by full dynamics for $10 \mathrm{ps}$. The substrate temperature was kept at $T=100 \mathrm{~K}$ again, which leads to the rapid cooling. After $10 \mathrm{ps,} \mathrm{the} \mathrm{tem-}$ perature of the film (melt) decreased into the region at around $100-200 \mathrm{~K}$. We named the final structure e $1 T 100 L Q$, according to our previous notations ( $Q$ is for 'quenching'). This technique can be 
considered as the computer simulation of splat cooling, where small droplets of melt are brought into contact with the chill-block. We performed this kind of simulation to retrieve information on the difference between rapid cooling and atom-byatom deposition on a substrate.

\section{Results}

Comparing models e1T100L and e1T100LQ, there is not really a significant difference in the statistical characteristics. However, locally the structures are not the same. In the e1T100L, there is a fourfold ring close to the diamond substrate (see Table 1 and Fig. 1; colored versions of our models can be seen on the following internet site: http://www.physik.uni-marburg.de/ kohary/ icams19.html). The ring is close to the substrate and every atom in the ring has $Z=4(Z$, is the coordination number). In model e $1 T 100 L Q$, this fourfold ring disappears and only fivefold, sixfold and sevenfold rings are present. Considering the ring statistics (Table 1), does not exist a big difference between these two models, except for the absence of the fourfold ring in e $1 T 100 L Q$. We note here that in amorphous silicon films [22], there is a surprising difference in the ring statistics between the models prepared by 'splat cooling' and by atom-by-atom deposited network. The ratio of the $\mathrm{sp}^{1}, \mathrm{sp}^{2}$ and $\mathrm{sp}^{3}$ sites obtained are almost the same in our constructed structures. Only one extra atom with $Z=4$ appeared in the e $1 T 100 L Q$ film, compared to the e1T100L model. The number of

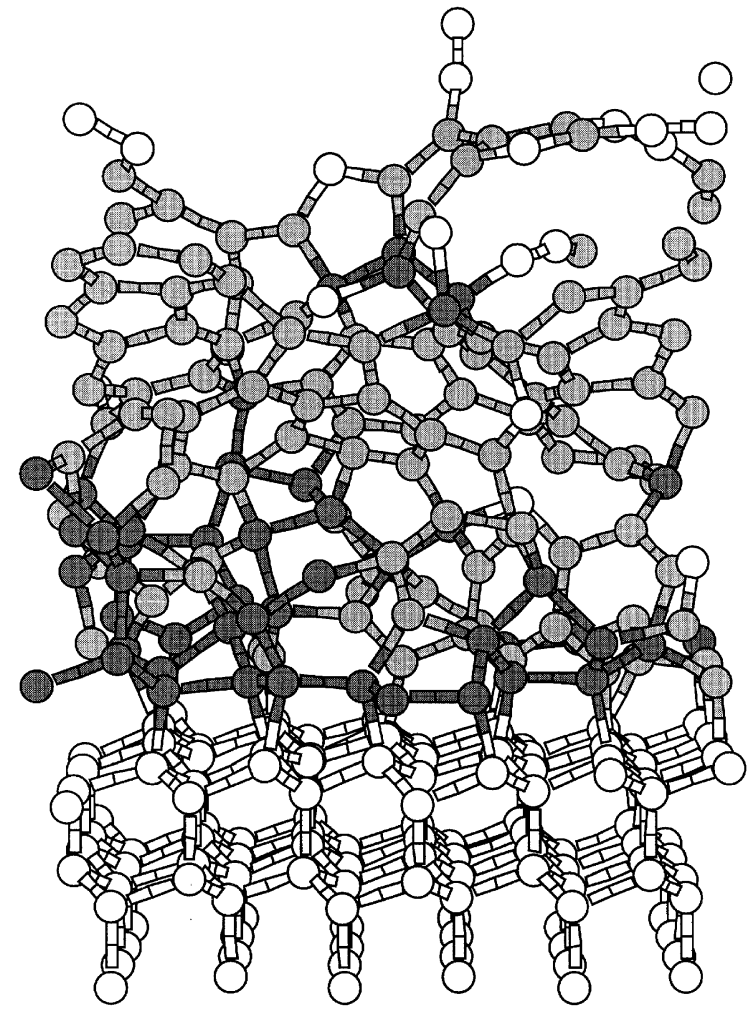

Fig. 1. Snapshot of model e $1 T 100 L$ is shown after growth and relaxation. The substrate (open circles at the bottom) temperature is $T_{\text {sub }}=100 \mathrm{~K}$. Black and grey atoms are fourfold- and threefold-coordinated. The open circles correspond to twofoldand one onefold-coordinated atoms. For a color version, please visit the http://www.physik.uni-marburg.de/ Rohary/icams19. html internet site.

atoms with a coordination number equal to three decreased by two and it increased by two for $Z=$

Table 1

Average bond and partial bond distances and their deviations (rms) and ring statistics for different models

\begin{tabular}{|c|c|c|c|c|c|c|c|c|c|c|c|c|}
\hline \multirow[t]{2}{*}{ Model } & \multicolumn{8}{|c|}{$\mathrm{C}_{i}-\mathrm{C}_{j}$ distance $(\AA)$} & \multicolumn{4}{|c|}{ Rings } \\
\hline & $\mathrm{C}-\mathrm{C}$ & $\mathrm{rms}$ & $\mathrm{C}_{3}-\mathrm{C}_{3}$ & $\mathrm{rms}$ & $\mathrm{C}_{3}-\mathrm{C}_{4}$ & $\mathrm{rms}$ & $\mathrm{C}_{4}-\mathrm{C}_{4}$ & $\mathrm{rms}$ & 4 & 5 & 6 & 7 \\
\hline e1T100 & 1.51 & 0.09 & 1.41 & 0.04 & 1.53 & 0.05 & 1.60 & 0.07 & 1 & 21 & 40 & 37 \\
\hline e $5 T 100$ & 1.50 & 0.09 & 1.44 & 0.05 & 1.53 & 0.05 & 1.58 & 0.06 & 0 & 26 & 43 & 42 \\
\hline e1T300 & 1.48 & 0.09 & 1.43 & 0.05 & 1.54 & 0.06 & 1.58 & 0.09 & 0 & 25 & 21 & 17 \\
\hline e $5 T 300$ & 1.50 & 0.09 & 1.43 & 0.05 & 1.54 & 0.06 & 1.58 & 0.06 & 0 & 18 & 32 & 20 \\
\hline $\mathrm{e} 1 T 100 L$ & 1.49 & 0.09 & 1.43 & 0.05 & 1.52 & 0.05 & 1.59 & 0.07 & 1 & 31 & 50 & 40 \\
\hline $\mathrm{e} 5 T 100 L$ & 1.52 & 0.09 & 1.44 & 0.06 & 1.54 & 0.06 & 1.59 & 0.06 & 0 & 44 & 54 & 58 \\
\hline e $1 T 100 L Q$ & 1.49 & 0.09 & 1.43 & 0.05 & 1.52 & 0.05 & 1.59 & 0.07 & 0 & 33 & 52 & 41 \\
\hline
\end{tabular}

The data belong to the bulk of the amorphous samples. (By our definition, the bulk is part of the amorphous structure over the substrate, apart from the $3 \AA$ thin layer on the top.) 
2. There is no carbon atom with $Z=5$ in our films. The densities of the bulks are almost the same, around $2.34 \mathrm{~g} / \mathrm{cm}^{3}$. Small differences can be observed in the partial bond distance and partial bond angle statistics.

Relaxations of the structures are more interesting. For the e1T100L model, the characteristics of the relaxation was described in [21]. In that paper, after the growth process the e1T100L film relaxed with full dynamics for 5 ps. During this period the substrate was kept at a constant temperature whilst the deposited network was cooling down. The decrease of the temperature was fitted according to $T_{\text {film }}=c+\exp (a t+b) \mathrm{K}$ previously. To find the best fit for the stretched exponential decrease $T(t)=100.0+\exp \left[a t^{\beta}+b\right] \mathrm{K}$, we prolonged the relaxation time for the e1T100 model by an additional $5 \mathrm{ps}$. The total relaxation for 10 ps can be seen in Fig. 2. The initial temperature of the amorphous film (melt) in e1T100LQ model was $4200 \mathrm{~K}$. It was then relaxed in a similar way, like the e1T100 model. Fig. 2 shows the temperature versus time for the latter case as well. The fitting data are $a=-2.66,-1.84 ; b=8.57,8.4$; $\beta=0.30,0.24$; for $\mathrm{e} 1 T 100$ and $\mathrm{e} 1 T 100 L Q$, respectively.

In Fig. 3 the comparison of bond angle deviation for e1T100 and e1T100LQ models can be seen, computed by the formula

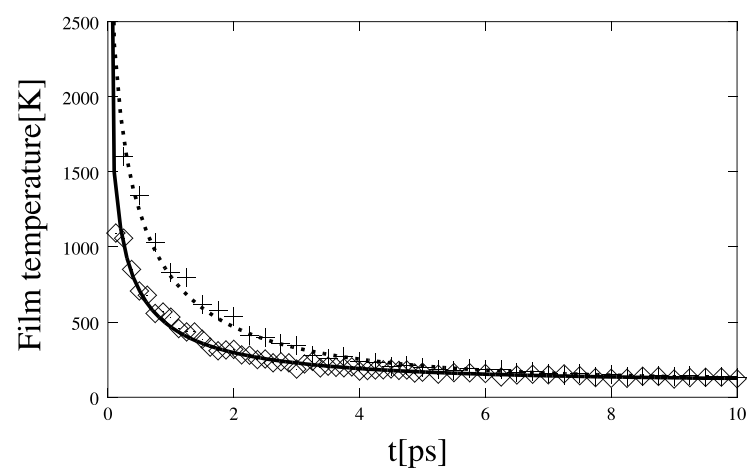

Fig. 2. Temperature versus time function during relaxation of models e1T100 (diamonds) and e $1 T 100 L Q$ (crosses). The straight and dotted lines are the fits according to $T(t)=100.0+$ $\exp \left[a t^{\beta}+b\right] \mathrm{K}$.

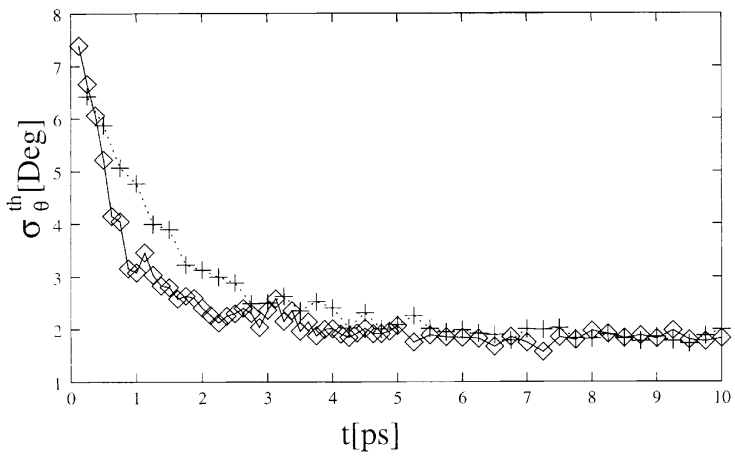

Fig. 3. As can be seen an expected $\sigma_{\Theta}^{\text {th }}$ versus time function has an exponential decay. Diamonds represent the dependence for e1T100 and crosses for e1 $T 100 L Q$.

$\sigma_{\Theta}^{\mathrm{th}}\left(t_{k}\right)=\sqrt{\frac{1}{N} \sum_{i}^{N}\left(\Theta_{i}\left(t_{k}\right)-\left\langle\Theta_{i}\right\rangle\right)^{2}}$,

where the time average $\left\langle\Theta_{i}\right\rangle$ of the bond angle $\Theta_{i}$ is used instead of $\Theta_{i}(T=0)$. All diagrams plotted show stretched-exponential behavior. More interestingly, if we plot the same deviations against the temperature of the amorphous film, we find almost linear dependence (see Fig. 4).

The radial distributions of the e $1 T 100 \mathrm{~L}$ model before and after relaxations are displayed in Fig. 5. The solid line shows the first and second neighbor contributions to radial distribution function (RDF) before relaxation, while the dotted line represents these contributions after relaxation. Peaks in the

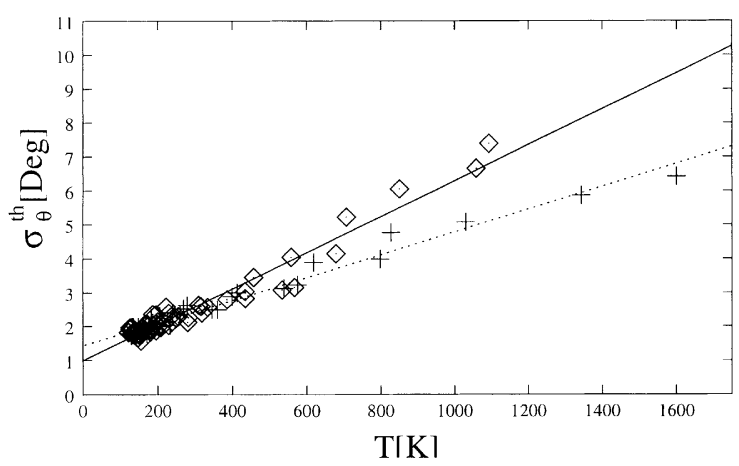

Fig. 4. The $\sigma_{\Theta}^{\text {th }}$ in function of temperature shows a linear relationship. Diamonds show the dependence for e1T100 and crosses for e1 $T 100 L Q$. Straight and dotted lines are fits of these points, respectively. Lines do not intersect the origin because usually $\left\langle\Theta_{i}\right\rangle \neq \Theta_{i}(T=0)$. 


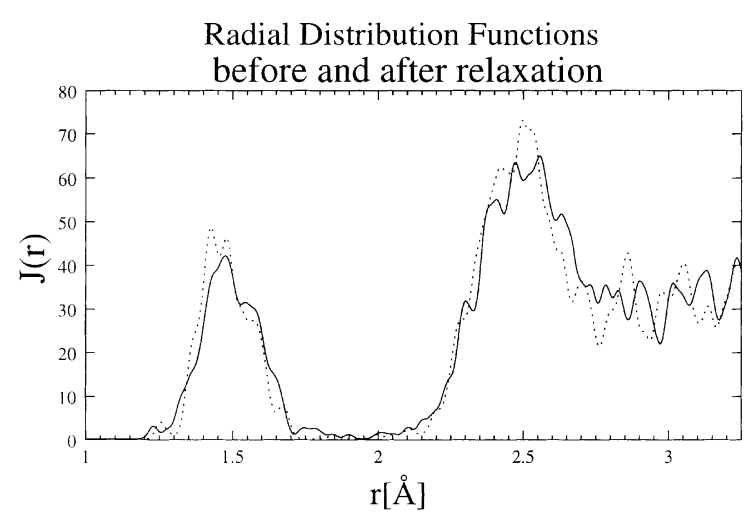

Fig. 5. Two radial distribution functions (RDFs) of model e1T100L are displayed here. The solid line shows the first and second neighbors contributions to RDF before relaxation, while dotted line shows these contributions after relaxation. First and second neighbor peaks in the final structure are a bit narrower.

final structure seem to be a bit narrower and the number of bonds having 1.6-2.2 $\AA$ bond lengths is less after relaxation. Numerical analysis provides the same conclusion.

\section{Discussion}

Here we turn back to the relaxation of amorphous film temperature against time, in Fig. 2. It can be seen that the two curves have different relaxation times and parameter $\beta$. We note here that the search for the best fit is not a well-defined problem in our case. A slight modification in the parameter list can give qualitatively almost the same fit. However, the difference between the two curves is conspicuous. The system size of the e1T100 model is smaller than for e1 $T 100 L Q$, and for this reason, the relaxation time of the latter is slower. For smaller systems, because of the temperature gradient in the $z$-direction, this kinetic energy loss is quicker, while for larger systems it is slower. We believe that this causes the description of the temperature decrease by a stretched-relaxation formula, but still a reasonable microscopic theory is needed to explain this behavior.

The similar dependence on temperature of the thermal deviations for bond angles, determined by Eq. (1), are shown in Fig. 3. Because the relaxation time is slower for larger systems, we see different slopes if we plot the same data of Fig. 3 against temperature (see Fig. 4). However, data for e1T100 and e1 $T 100 L Q$ seem to coincide at low temperatures in Fig. 4 and would provide almost the same deviations from average bond distances and angles at zero temperature, caused simply by the fact of amorphous disorder.

\section{Conclusions}

In summary, low energy MD simulations of atomic beam growth on a diamond [1 111$]$ surface were carried out. Six different networks were grown by atom-by-atom deposition techniques and the seventh structure was made by a 'splat cooling' technique. The latter method is similar to melt quenching methods, which are widely applied in computer simulations to generate the bulk of amorphous networks. After the growth, the structures were relaxed. The atoms could move with full dynamics in the amorphous film over the substrate, and the substrate temperature was kept constant. The temperature relaxations show stretched-exponential functions. The model prepared by the 'splat cooling' method is not different in a statistical sense to the deposited networks. Our quantum mechanical treatment of the interatomic potential for describing the growth of a-C films is between the pioneer works in this field. The growth process was described as in real experiments without any artificial model of energy dissipation [18].

\section{Acknowledgements}

This work has been supported by the Fund OTKA (Grant No. T032193, T033004). We would like to thank Dr I. László for his contribution in developing the computer codes.

\section{References}

[1] P.H. Gaskell, A. Saeed, P. Chieux, D.R. McKenzie, Phys. Rev. Lett. 67 (1991) 1286.

[2] S. Kugler, K. Shimakawa, T. Watanabe, K. Hayashi, I. László, R. Bellissent, J. Non-Cryst. Solids 164-166 (1993) 831. 
[3] P.C. Kelires, Phys. Rev. B 62 (2000) 15686.

[4] J.K. Walters, K.W.R. Gilkes, J.D. Wicks, R.J. Newport, Phys. Rev. B 58 (1998) 8267.

[5] H.-P. Kaukonen, R.M. Nieminen, Phys. Rev. Lett. 68 (1992) 620; Phys. Rev. B 61 (2000) 2806.

[6] H.U. Jäger, K. Albe, J. Appl. Phys. 88 (2000) 1129.

[7] J. Tersoff, Phys. Rev. Lett. 61 (1988) 2879.

[8] D.W. Brenner, Phys. Rev. B 42 (1990) 9458.

[9] R. Car, M. Parrinello, Phys. Rev. Lett. 55 (1985) 2471.

[10] G. Galli, R.M. Martin, R. Car, M. Parrinello, Phys. Rev. Lett. 62 (1989) 555.

[11] N.A. Marks, D.R. McKenzie, B.A. Pailthorpe, M. Bernasconi, M. Parrinello, Phys. Rev. B 54 (1996) 9703.

[12] D.G. McCulloch, D.R. McKenzie, C.M. Goringe, Phys. Rev. B 61 (2000) 2349.
[13] C.Z. Wang, K.M. Ho, C.T. Chan, Phys. Rev. Lett. 70 (1993) 611.

[14] D.A. Drabold, P.A. Fedders, P. Stumm, Phys. Rev. B. 49 (1994) 16415.

[15] D. Porezag, Th. Frauenheim, Th. Koehler, G. Seifert, R. Kaschner, Phys. Rev. B 51 (1995) 12947.

[16] J. Dong, D.A. Drabold, Phys. Rev. B. 57 (1998) 15591.

[17] S. Uhlmann, Th. Frauenheim, Y. Lifshitz, Phys. Rev. Lett. 81 (1998) 641.

[18] K. Kohary, S. Kugler, J. Non-Cryst. Solids 266-269 (2000) 746.

[19] C.H. Xu, C.Z. Wang, C.T. Chan, K.M. Ho, J. Phys. Condens. Matter 4 (1992) 6047.

[20] I. László, Europhys. Lett. 44 (1998) 741;

J. Mol. Struct. (THEOCHEM) 463 (1999) 181.

[21] K. Kohary, S. Kugler, Phys. Rev. B 63 (2001) 193404.

[22] K. Kohary, S. Kugler, to be published. 